10

\title{
Термомеханика деформаций в ангармоническом твердом теле
}

\author{
() Н.Н. Горобей, А.С. Лукьяненко
}

Санкт-Петербургский политехнический университет Петра Великого

Санкт-Петербург, Россия

E-mail: n.gorobey@mail.ru

(Поступила в Редакцию 29 октября 2018 г.

В окончательной редакции 29 октября 2018 г.

Принята к публикации 7 ноября 2018 г.)

\begin{abstract}
Макроскопические законы, определяющие температуру и деформации ангармонического твердого тела в заданном внешнем температурно-силовом поле, сформулированы в виде первого начала термодинамики, дополненного уравнениями состояния тела. Необходимые для этого внутренняя и свободная энергии, находятся из статистической суммы, в которой часть степеней свободы тела, определяющих его форму, освобождены от статистического усреднения. Эти функции состояния вычислены с точностью до первого порядка теории возмущений по ангармонизму для микроскопической динамической модели тела с заданной в виде ряда по степеням координат атомов потенциальной энергией межатомного взаимодействия. Рассматривается классическая область высоких температур.
\end{abstract}

DOI: 10.21883/FTT.2019.04.47427.295

\section{1. Введение}

К тепловым явлениям, связанным с механическими деформациями в ангармоническом твердом теле, относятся тепловое расширение [1] и термоупругий эффект [2,3]. Последний заключается в небольшом изменении температуры тела при его адиабатическом механическом деформировании. Термодинамический аспект этого явления обсуждается в работах $[4,5,6]$. Тесная связь механической деформации с температурой тела оправдывает использование термина „термомеханика“ для описания этих явлений на макроскопическом уровне. Это подразумевает, что одной частью этого описания является первое начало термодинамики, а механический аспект целиком заключен в уравнении состояния тела. Для установления связи между теплотой и механикой следует обратиться на микроскопический уровень. В случае одного ангармонического осциллятора [7] она заключена в адиабатическом инварианте

$$
I=\frac{E}{\omega},
$$

где $E$ - энергия колебаний, а $\omega$ - частота осциллятора. При деформировании ангармонического твердого тела частоты колебаний атомов изменяются (эффект Грюнайзена), что вызывает изменение колебательной энергии. В работе [6] связь деформации и температуры для одномерной цепочки атомов установлена с использованием так называемого самосогласованного гармонического приближения. В работе [8] для вычисления статистической суммы и основных функций состояния ансамбля ангармонических осцилляторов внутренней и свободной энергий используется теория возмущений по константе ангармонизма. При этом среднее удлинение осциллятора в большом ансамбле, будучи одной из степеней свободы системы, рассматривается как макроскопический параметр, который освобождается от статистического усреднения и должен определяться условием механического равновесия на макроуровне. В данной работе этот подход сформулирован в общем виде для произвольного твердого тела. Параметры формы твердого тела, наравне с его температурой, рассматриваются в качестве параметров состояния. Само состояние определяется функциями этих параметров - внутренней и свободной энергией и их значениями. В данной работе рассматривается высокотемпературное приближение, не учитывающее квантовые эффекты.

В следующем разделе в рамках ограниченного статистического усреднения, при фиксированных параметрах формы поверхности, получены формулы для функций состояния и сформулированы два основных закона термомеханики - первое начало термодинамики и уравнения состояния как условия механического равновесия поверхности во внешнем температурно-силовом поле. Во втором разделе функции состояния вычисляются с точностью до первого порядка по константам кубического ангармонизма.

\section{2. Статистическая сумма и основные термодинамические функции}

Микроскопическая структура твердого тела полностью определяется потенциальной энергией взаимодействия его атомов. При этом мы считаем, что атомы своим расположением образуют стабильную пространственную структуру (в рассматриваемом интер- 
вале температур), так что структурные фазовые переходы исключаются. Разделим атомы на два класса объемные (bulk) и поверхностные (surface). Сообразно этому представим потенциальную энергию межатомного взаимодействия тела в виде суммы

$$
U\left(x_{\beta i}, x_{\sigma s}\right)=U_{b}\left(x_{\beta i}\right)+U_{s}\left(x_{\sigma s}\right)+U_{i n t}\left(x_{\beta i}, x_{\sigma s}\right),
$$

где каждое слагаемое представляет собой ряд по степеням координат атомов, причем учитываются поправки, выходящие за рамки гармонического приближения [9]. Здесь используются следующие обозначения: $x_{\beta i}-$ декартовы координаты объемных атомов (начало отсчета помещаем в центр масс тела), $\beta=1,2, \ldots, N_{b}-$ их нумерующий индекс, $x_{\sigma s}$ - декартовы координаты атомов поверхности, $\sigma=1,2, \ldots, N_{s}-$ их нумерующий индекс. Все три слагаемых в (2) в общем случае представляются степенными рядами по этим координатам. Для части взаимодействия мы ограничимся здесь приближением кубического ангармонизма и запишем:

$$
\begin{aligned}
U_{i n t}\left(x_{\beta i}, x_{\sigma s}\right)= & c_{\beta i \sigma s} x_{\beta i} x_{\sigma s}-h_{\beta i \beta^{\prime} i^{\prime} \sigma s} x_{\beta i} x_{\beta^{\prime} i^{\prime}} x_{\sigma-s} \\
& +d_{\beta i \sigma s \sigma^{\prime} s^{\prime}} x_{\beta i} x_{\sigma s} x_{\sigma^{\prime} s^{\prime}} .
\end{aligned}
$$

Здесь по повторяющимся индексам предполагается суммирование. Первое слагаемое в (3) - гармоническая часть взаимодействия, а два последних - ангармоническая.

Координаты атомов поверхности $x_{\sigma s}$ будут служить далее параметрами формы тела. Они, хотя и совершают термодинамические флуктуации, имеют гораздо большие макроскопические сдвиги при тепловом и механическом воздействии на тело. В данной работе ограничимся рассмотрением поверхностных внешних сил $F_{\sigma s}$, которые имеют точками приложения атомы поверхности. Считаем, что результирующая этих сил и их суммарный момент равны нулю

$$
\begin{gathered}
\sum_{\sigma} F_{\sigma s}=0, \\
\varphi_{s} \equiv \sum_{\sigma} \varepsilon_{s t u} x_{\sigma t} F_{\sigma u}=0 .
\end{gathered}
$$

Здесь $\varepsilon_{s t u}$ - полностью антисимметричный тензор. Запишем статистическую сумму

$$
Z=\int \prod_{\beta i} d p_{\beta i} \int \prod_{\beta i} d x_{\beta i} \exp \left[-\frac{H\left(p_{\beta i} x_{\beta i} x_{\sigma s}\right)}{k T}\right],
$$

где функция Гамильтона имеет вид

$$
H\left(p_{\beta i} x_{\beta i} x_{\sigma s}\right)=\sum_{\beta i} \frac{p_{\beta i}^{2}}{2 m_{\beta}}+U\left(x_{\beta i}, x_{\sigma s}\right) .
$$

Следуя [8], мы исключаем динамику атомов поверхности из статистического усреднения. После этого две основные для нашего рассмотрения функции состояния определяются уравнениями [10]

$$
E\left(T, x_{\sigma s}\right)=\frac{\partial}{\partial \beta} \ln Z
$$

— внутренняя энергия, и

$$
H\left(T, x_{\sigma s}\right)=-\frac{1}{\beta} \ln Z,
$$

- свободная энергия, где $\beta \equiv 1 / k T$.

С помощью этих функций состояния сформулируем два основных закона термомеханики твердого тела, которые определяют параметры его состояния $T, x_{\sigma s}$ при тепловом и механическом воздействии. Первое начало термодинамики запишем в дифференциальной форме:

$$
d Q=d E+d A
$$

где $d Q-$ количество переданного телу тепла, а

$$
d A=\sum_{\sigma s} F_{\sigma s} d x_{\sigma s}
$$

- работа по изменению формы поверхности во внешнем силовом поле.

Уравнение (10) определяет изменение температуры тела при рассматриваемых тепловом и механическом воздействиях. В частности, при адиабатическом механическом деформировании следует положить $d Q=0$.

Второй закон - условия механического равновесия формы поверхности при заданной температуре $T$ и заданных внешних силах $F_{\sigma s}$. Его следует формулировать, с учетом (5), следующим образом:

$$
\frac{\partial H}{\partial x_{\sigma s}}+\sum_{t} \lambda_{t} \frac{\partial \varphi_{t}}{\partial x_{\sigma s}}=F_{\sigma s}
$$

где $\lambda_{t}$ - неопределенные множители Лагранжа, которые находятся затем из условия (5).

\section{3. Законы термомеханики в первом порядке теории возмущений}

Продемонстрируем к чему сводится вычисление статистической суммы в рамках теории возмущений. Ограничимся здесь приближением кубического ангармонизма также для потенциальной энергии объема тела

$$
U_{b}\left(x_{\beta i}\right)=\frac{1}{2} f_{\beta i \beta^{\prime} i^{\prime}} x_{\beta i} x_{\beta^{\prime} i^{\prime}}-\frac{1}{3} g_{\beta i \beta^{\prime} i^{\prime} \beta^{\prime \prime} i^{\prime \prime}} x_{\beta^{\prime} i^{\prime}} x_{\beta^{\prime \prime} i^{\prime \prime}} .
$$

Полную потенциальную энергию, с учетом (3), запишем в виде

$$
\begin{aligned}
U\left(x_{\beta i}, x_{\sigma s}\right)= & \frac{1}{2} A_{\beta i \beta^{\prime} i^{\prime}} x_{\beta i} x_{\beta^{\prime} i^{\prime}}+b_{\beta i} x_{\beta i}+U_{[S]}\left(x_{\sigma s}\right) \\
& -\frac{1}{3} g_{\beta i \beta^{\prime} i^{\prime} \beta^{\prime \prime} i^{\prime \prime}} x_{\beta i} x_{\beta^{\prime} i^{\prime}} x_{\beta^{\prime \prime} i^{\prime \prime}},
\end{aligned}
$$


где обозначено

$$
A_{\beta i \beta^{\prime} i^{\prime}} \equiv f_{\beta i \beta^{\prime} i^{\prime}}-2 h_{\beta i \beta^{\prime} i^{\prime} \sigma s} x_{\sigma s}
$$

— матрица „смягченных“ ангармонизмом силовых постоянных,

$$
b_{\beta i} \equiv c_{\beta i \sigma s} x_{\sigma s}+d_{\beta i \sigma s \sigma^{\prime} s^{\prime}} x_{\sigma s} x_{\sigma^{\prime} s^{\prime}} .
$$

Займемся теперь вычислением статистической суммы (6). Интеграл по импульсам дает множитель

$$
(2 \pi k T)^{3 N_{b} / 2} \prod_{\beta i} \sqrt{m_{\beta}} .
$$

Интеграл по координатам вычислим с точностью до первого порядка по константам ангармонизма $g_{\beta i \beta^{\prime} i^{\prime} \beta^{\prime \prime} i^{\prime \prime}}$.

Это сводится к вычислению гауссова интеграла

$$
\begin{aligned}
\int \prod_{\beta i} d x_{\beta i} & \exp \left(-\frac{1}{2 k T} A_{\beta i \beta^{\prime} i^{\prime}} x_{\beta i} x_{\beta^{\prime} i^{\prime}}-\frac{b_{\beta i} x_{\beta i}}{k T}\right) \\
\times & \left(1-\frac{1}{3 k T} g_{\beta i \beta^{\prime} i^{\prime} \beta^{\prime \prime} i^{\prime \prime}} x_{\beta i} x_{\beta^{\prime} i^{\prime}} x_{\beta^{\prime \prime} i^{\prime \prime}}\right)
\end{aligned}
$$

и введения на промежуточном этапе новых переменных интегрирования

$$
u_{\beta i}=x_{\beta i}-\left(A^{-1}\right)_{\beta i \alpha k} b_{\alpha k} .
$$

Результат вычисления

$$
\begin{aligned}
& \frac{(2 \pi k T)^{3 N_{b} / 2}}{\sqrt{\operatorname{det} A}} \exp \left[-\frac{1}{2 k T}\left(A^{-1}\right)_{\alpha k \alpha^{\prime} k^{\prime}} b_{\alpha k} b_{\alpha^{\prime} k^{\prime}}\right] \\
& \times\left\{1-\frac{1}{3 k T} g_{\beta i \beta^{\prime} i^{\prime} \beta^{\prime \prime} i^{\prime \prime}}\left[\frac{3}{2} k T\left(A^{-1}\right)_{\beta i \beta^{\prime} i^{\prime}}\left(A^{-1}\right)_{\beta^{\prime \prime} i^{\prime \prime} \alpha k} b_{\alpha k}\right.\right. \\
& \left.\left.\quad+\left(A^{-1}\right)_{\beta i \alpha k}\left(A^{-1}\right)_{\beta^{\prime} i^{\prime} \alpha^{\prime} k^{\prime}}\left(A^{-1}\right)_{\beta^{\prime \prime} i^{\prime \prime} \alpha^{\prime \prime} k^{\prime \prime}} b_{\alpha k} b_{\alpha^{\prime} k^{\prime}} b_{\alpha^{\prime \prime} k^{\prime \prime}}\right]\right\}
\end{aligned}
$$

После этого запишем с точностью до первого порядка

$$
\begin{aligned}
\ln Z & =\frac{3 N_{b}}{2} \ln k T-\frac{1}{2} \ln \operatorname{det} A \\
& -\frac{1}{2 k T}\left(A^{-1}\right)_{\alpha k \alpha^{\prime} k^{\prime}} b_{\alpha k} b_{\alpha^{\prime} k^{\prime}}-\frac{U_{s}\left(x_{\sigma s}\right)}{k T} \\
& -\frac{1}{3 k T} g_{\beta i \beta^{\prime} i^{\prime} \beta^{\prime \prime} i^{\prime \prime}}\left[\frac{3}{2} k T\left(A^{-1}\right)_{\beta i \beta^{\prime} i^{\prime}}\left(A^{-1}\right)_{\beta^{\prime \prime} i^{\prime \prime} \alpha k} b_{\alpha k}\right. \\
& \left.+\left(A^{-1}\right)_{\beta i \alpha k}\left(A^{-1}\right)_{\beta^{\prime} i^{\prime} \alpha^{\prime} k^{\prime}}\left(A^{-1}\right)_{\beta^{\prime \prime} i^{\prime \prime} \alpha^{\prime \prime} k^{\prime \prime}} b_{\alpha k} b_{\alpha^{\prime} k^{\prime}} b_{\alpha^{\prime \prime} k^{\prime \prime}}\right] .
\end{aligned}
$$

При этом мы отбросили несущественную для нас константу

$$
3 N_{b} \ln 2 \pi+3 \sum_{\beta} \ln m_{\beta} .
$$

Отсюда получаем внутреннюю энергиюс точностью до первого порядка по константам ангармонизма $g_{\beta i \beta^{\prime} i^{\prime} \beta^{\prime \prime} i^{\prime \prime}}$

$$
\begin{aligned}
& E\left(T, x_{\sigma s}\right)=3 N_{b} k T+U_{s}\left(x_{\sigma s}\right) \\
& \quad+\frac{1}{2}\left(A^{-1}\right)_{\alpha k \alpha^{\prime} k^{\prime}} b_{\alpha k} b_{\alpha^{\prime} k^{\prime}}+\frac{1}{3} g_{\beta i \beta^{\prime} i^{\prime} \beta^{\prime \prime} i^{\prime \prime}}\left(A^{-1}\right)_{\beta i \alpha k} \\
& \quad \times\left(A^{-1}\right)_{\beta^{\prime} i^{\prime} \alpha^{\prime} k^{\prime}}\left(A^{-1}\right)_{\beta^{\prime \prime} i^{\prime \prime} \alpha^{\prime \prime} k^{\prime \prime}} b_{\alpha k} b_{\alpha^{\prime} k^{\prime}{ }^{\prime}} b_{\alpha^{\prime \prime} k^{\prime \prime}} .
\end{aligned}
$$

$\mathrm{C}$ той же точностью внутренняя энергия равна

$$
\begin{aligned}
H\left(T, x_{\sigma s}\right) & =\frac{k T}{2} \ln \operatorname{det} A+\frac{1}{2}\left(A^{-1}\right)_{\alpha k \alpha^{\prime} k^{\prime}} b_{\alpha k} b_{\alpha^{\prime} k^{\prime}}+U_{S}\left(x_{\sigma s}\right) \\
& +\frac{k T}{2} g_{\beta i \beta^{\prime} i^{\prime} \beta^{\prime \prime} i^{\prime \prime}}\left(A^{-1}\right)_{\beta i \beta^{\prime} i^{\prime}}\left(A^{-1}\right)_{\beta^{\prime \prime} i^{\prime \prime} \alpha k} b_{\alpha k} \\
& +\frac{1}{3} g_{\beta i \beta^{\prime} i^{\prime} \beta^{\prime \prime} i^{\prime \prime}}\left(A^{-1}\right)_{\beta i \alpha k}\left(A^{-1}\right)_{\beta^{\prime} i^{\prime} \alpha^{\prime} k^{\prime}} \\
& \times\left(A^{-1}\right)_{\beta^{\prime \prime} i^{\prime \prime} \alpha^{\prime \prime} k^{\prime \prime}} b_{\alpha k} b_{\alpha^{\prime} k^{\prime}} b_{\alpha^{\prime \prime} k^{\prime \prime}},
\end{aligned}
$$

где мы отбросили несущественный для условий механического равновесия вклад

$$
-\frac{3 N_{b} \ln k T}{2 k T} .
$$

Теперь есть все необходимое для формулировки законов термомеханики с точностью до первого порядка по константам ангармонизма. Здесь мы не будем выписывать эти законы в явном виде ввиду их громоздкости. Отметим только, что связь между деформацией поверхности и температурой тела заключена в первом и четвертом слагаемых в выражении (24) для свободной энергии. Оба эти слагаемых пропорциональны температуре и явно зависят от координат атомов поверхности $x_{\sigma s}$. В отсутствие ангармонизма, кода $h_{\beta i \beta^{\prime} i^{\prime} \sigma s}=g_{\beta i \beta^{\prime} i^{\prime} \beta^{\prime \prime} i^{\prime \prime}}=0$, тепловые и механические явления в теле не связаны между собой.

\section{4. Заключение}

Таким образом, макроскопические законы, определяющие температуру и деформации ангармоническоготвердого тела при его нагревании и механическом воздействии, сводятся к первому началу термодинамики, дополненному уравнениями состояния. В свою очередь, эти законы могут быть получены в результате вычисления статистической суммы для известной кристаллической структуры и потенциальной энергии межатомного взаимодействия при условии, что тело находится в состоянии термодинамического и механического равновесия в заданном внешнем силовом поле. При этом часть степеней свободы тела, определяющих форму его поверхности, считаются параметрами состояния и исключаются из статистического усреднения. Связь между температурой и деформациями тела возникает как следствие ангармонизма межатомных взаимодействий и полностью отражена в его свободной энергии.

Авторы благодарят А.В. Гольцева за полезные дискуссии. 


\section{Список литературы}

[1] Ч. Киттель. Введение в физику твердого тела. Гостехиздат, M. (1957). $524 \mathrm{c}$.

[2] J.P. Joule. Proc. R. Soc. 8, 564 (1857).

[3] W. Thompson (Lord Kelvin). Trans. Roy. Soc. Edinburgh 20, 261 (1853).

[4] R.T. Potter, L.J. Greeves. Proc. SPIE 817, 134 (1987).

[5] A.A. Benam, G. Viola, T. Korakianitis. J. Therm. Anal. Calorim. 100, 941 (2010).

[6] В.Л. Гиляров, А.И. Слуцкер, В.П. Володин, А.И. Лайус. ФТТ 40, 1548 (1998).

[7] A.I. Slutsker, V.P. Volodin. Thermochimia Acta 247, 111 (1994).

[8] Н.Н. Горобей, А.С. Лукьяненко. ФТТ 56, 2187 (2014).

[9] Solid State Physics. Supplement 3: Theory of Lattice Dynamics in the Harmonic Approximation / Eds A.A. Maradudin, E.W. Montroll, G.H. Weiss, I.P. Ipatova. Academic Press, London (1971).

[10] Л.Д. Ландау, Е.М. Лифшиц. Статистическая физика. Наука, М. (1976). Ч. $1.583 \mathrm{c}$.

Редактор Т.Н. Василевская 